\title{
Performance analysis of three kinds of non-coherent detectors for ultra-wideband Communications
}

\author{
Jian Pan*, Sheng Zhang, Benzhou Jin, Xiaokang Lin \\ Department of Electronic Engineering, Tsinghua University \\ Beijing, China \\ *Email: jianlonger@foxmail.com
}

\begin{abstract}
This paper discusses the basic signal detection scheme and error performances of three kind receiver architecture, i.e., the peak detector, the square-law detector and the direct-integral receiver. The theoretical bit error probabilities are derived through Gaussian approximation that is well satisfied in UWB systems. Simulations are performed in the additive white Gaussian (AWGN) channel model and results shows that the theoretical curve derived from the analytical model is in accordance with the experimental curve. This paper indicates that the bit error rate (BER) performance of direct-integral detectors is slightly better than energy detectors (EDs) when $E_{b} / N_{0}$ is small (less than 6.5db). Besides, this paper indicates that, compare with coherent detectors, Direct-integral detector has a potential to achieve an appropriate tradeoff between error rate performance and system complexity.
\end{abstract}

Keywords- Direct-integral receiver; quare-law detector; Trigger; ultra-wideband.

\section{INTRODUCTION}

Former UWB system designers mostly focused on using coherent architectures, typically Rake schemes, at the receiver end in order to achieve high data rate and good error rate performance. However, it has been claimed that the optimum receivers face many challenges in practical implementation due to UWB channel characteristics and signal spectral densities constrains[1][2].

The energy-detection-based UWB receiver architecture known as EDs has been attracting much attention recently because of its good performance on low-complexity, lowpower and low cost solutions for practical applications such as UWB wireless sensor networks. The ED receiver is consisted by an square-law detector which measures the energy in the received waveform over an observation time window, though this kind receiver has much merit, when we focus on low SNR(signal noise rate) condition, its performance is no better than an simple receiver we proposed which can be defined as direct-integral receiver. A direct-integral receiver is formed by a linear detector which measures the integral of received waveform over an observation time window. In this paper, we only discuss the performance of these receivers over no-fading, no-multipath channel, because in short distance $(0.1-1 \mathrm{~m})$ communicating, this assume is reasonable.

Our contribution in this paper is twofold. First, we offer the detailed deduce from the theoretical analysis and obtain expressions for the error probability of detection over AWGN channel. Second, and more importantly, the influence of decreasing observation time duration is discussed. The simulations show that, the ED receiver is inferior to direct-integral receiver in low SNR and superior to direct-integral receiver in high SNR.

The paper is organized as follows: The System models for the two receiver are described in Section II; Section III is devoted to deduce the expression of error performance for Direct-integral receiver; In Section IV, the BER performance for ED Receiver is discussed; In Section V, simulation results under different conditions are presented; Finally, comparisons are demonstrated and analyzed in Section VI, and Conclusions are offered in Section VII.

\section{SYSTEM MODELS}

While our analysis applies to either low-pass (LP) or band pass (BP) systems, we here focus on the LP representation. The received signal at the receiver end can be represented as

$$
r_{0}(t)=a_{i} \bullet s_{L}(t)+n(t) \quad a_{i} \in\{0,1\}
$$

Here, $a_{i}$ is decided by the sending symbol ' 1 ' and ' 0 '. $S_{L}(t)$ is the baseband signal, $\boldsymbol{n ( t )}$ is an AWGN process with a zero mean and a known flat power spectral density (PSD). Let $N_{0}$ denote the one-sided noise PSD and $B$ the signal bandwidth (i.e., positive spectrum support).

Fig.1. shows the proposed direct-integral receiver architecture. The received signal is first prefiltered by an ideal $L P$ filter. Then, the output of this filter is integrated over a time interval $T_{c}$ to finally produce a measure of the electric quantity of the received waveform. Fig.2. shows the peak detector, which employ the peak of received waveform to trigger the sampler and utilize this value for symbol decision. Fig.3. refers to the ED receiver.

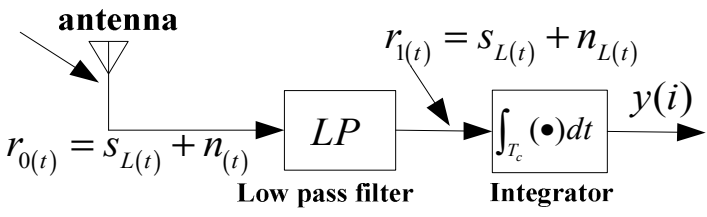

Fig.1 Architecture of Direct-integral receiver 


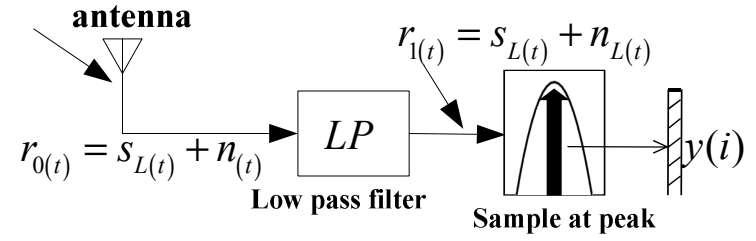

Fig. 2 Architecture of peak detector

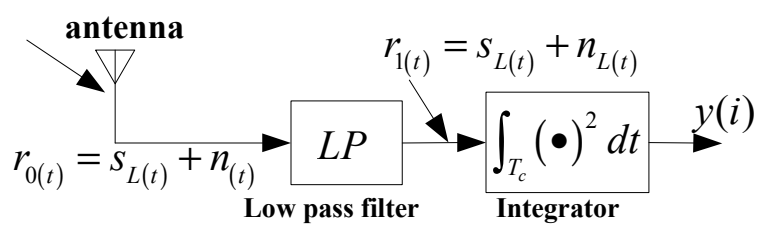

Fig. 3 Architecture of Direct-integral receiver

\section{Detection AND ERror RAte Probabilities Over AWGN CHANNELS For THREE KIND RECEIVER}

\section{A. BER for Direct-integral receiver}

As shown in Fig.1. $r_{I}(t)$ denotes the demodulated signal of $r_{0}(t)$, and as the figure shows, the bandwidth of $L P$ is identical to the efficient bandwidth of signal $S_{L}(t)$, hence the filter outputs the whole signal while strains the noise beyond its passband. Thus the noise output is $n_{L}(\mathrm{t})$, which is a gauss process with Zero mean its mean 0 and variance of $B N_{0}$.

The autocorrelation of $n_{L}(t)$ can be expressed as

$$
R_{n}(\tau)=\frac{N_{0}}{2} \bullet \frac{\sin (\pi B \tau)}{\pi}=N_{0} B \bullet \sin c(\pi B \tau)
$$

The output of integrator denoted by $y$ acts as a test statistic to test the two hypotheses $H_{1}$ (when sending symbol ' 1 ') and $H_{0}$ (when sending symbol ' 0 '). Thus $y$ can be expressed as

$$
\begin{gathered}
y=\int_{0}^{T_{c}} a_{i} \bullet s_{L(t)}+n_{L(t)} d t \\
m_{0}=E\left(y / H_{0}\right)=0 \\
m_{1}=E\left(y / H_{1}\right)=\int_{0}^{T_{c}} s_{L(t)} d t \approx \Delta \sum_{i=1}^{M} s_{L}(i \Delta) \\
\sigma^{2}=\operatorname{var}\left(y / H_{1}\right)=\int_{0}^{T_{c} T_{c}} \int_{0} E\left[n_{L\left(t_{1}\right)} n_{L\left(t_{2}\right)}\right] d t_{1} d t_{2} \\
=\int_{0}^{T_{c} T_{c}} R_{0}\left(t_{1}-t_{2}\right) d t_{1} d t_{2}=\frac{N_{0}}{2} \int_{0}^{T_{c}} \int_{0}^{T_{c}} \frac{\sin \pi B\left(t_{1}-t_{2}\right)}{\pi\left(t_{1}-t_{2}\right)} d t_{1} d t_{2} \\
=N_{0} B \int_{0} \int_{0} \sin c\left[\pi B\left(t_{1}-t_{2}\right)\right] d t_{1} d t_{2}
\end{gathered}
$$

Where $M$ is the number of samples in per code pulse (we finally have terms to sum over), $T_{\mathrm{s}}=T_{\mathrm{c}} / M$ is the sample interval. Then under $H_{l}, y$ follows a non-central gauss distribution with variance $\sigma^{2}$, and $m_{l}$ as its mean. Now, the probability density function (PDF) of $y$ can be expressed as

$$
f_{Y}(y)= \begin{cases}\frac{1}{2 \pi \sigma} \exp \left(-\frac{y^{2}}{2 \sigma^{2}}\right) & H_{0} \\ \frac{1}{2 \pi \sigma} \exp \left[-\frac{\left(y-m_{1}\right)^{2}}{2 \sigma^{2}}\right] & H_{1}\end{cases}
$$

Under the Gaussian approximation, error performances for OOK and 2-PPM systems can be derived since the mean and variance of $Y$ is known.

In OOK, there are two frame errors:

i) Miss alarm error: It means no pulse is detected when a pulse frame is transmitted.

ii) False alarm error: This happens when an empty frame is transmitted but a pulse is detected.

Using the statistical characteristics of $Y$, we have

$$
\begin{gathered}
P_{1 \mid 0}=\frac{1}{\sqrt{2 \pi} \sigma} \int_{V}^{\infty} e^{-x^{2} / 2 \sigma^{2}} d x \\
P_{0 \mid 1}=\frac{1}{\sqrt{2 \pi} \sigma} \int_{-\infty}^{V} e^{\left(x-m_{1}\right)^{2} / 2 \sigma^{2}} d x
\end{gathered}
$$

Where, $P_{1 \mid 0}$ and $P_{0 \mid 1}$ represent the false alarm error probabil-ity and the Leak-detecting error probability respectively. $m_{1}$, see expression (5), is the mean of $Y$ when the binary symbol $a_{\mathrm{i}}$, and $\sigma^{2}$ is the variance of $Y$. In order to obtain the best error performance, the optimum threshold $V$ can be solved as $V=m_{1} / 2$.

Then, the bit error probability for OOK is

$$
\begin{aligned}
P_{e 1_{-} \text {OOK }} & =\frac{1}{2} P_{1 \mid 0}+\frac{1}{2} P_{0 \mid 1}=Q\left(\frac{m_{1}-V}{\sigma}\right) \\
& =Q\left(\frac{m_{1}}{2 \sigma}\right)
\end{aligned}
$$

Since a maximum likelihood decoder can be adopted in 2PPM systems, it requires no threshold.

$$
P_{e 1_{-} P P M}=p\left\{m_{1}+n_{1}<n_{2}\right\}
$$

Where, $m_{1}$ is the same as in equation (5). $n_{1}, n_{2}$ is given as follow

$$
\begin{gathered}
n_{1}=\int_{0}^{T_{c}} n_{L(t)} d t \quad n_{2}=\int_{T_{c}}^{2 T_{c}} n_{L(t)} d t \\
\operatorname{cov}\left(n_{1}, n_{2}\right)=E\left[n_{1} n_{2}\right]=E\left[\int_{T_{c}}^{2 T_{c}} \int_{0}^{T_{c}} n_{L\left(t_{1}\right)} n_{L\left(t_{2}\right)} d t_{1} d t_{2}\right] \\
=\int_{T_{c}}^{2 T_{c} T_{c}} \int_{0}^{2 T_{c}} R_{n}\left(t_{1}-t_{2}\right) d t_{1} d t_{2}=N_{0} B \int_{T_{c}}^{T_{c}} \sin c\left[\pi B\left(t_{1}-t_{2}\right)\right] d t_{1} d t_{2}
\end{gathered}
$$

The bit error probability can be easily derived as 


$$
\begin{aligned}
P_{e 1_{-} P P M} & =p\left\{m_{1}+n_{1}<n_{2}\right\} \\
& =Q\left(\frac{m_{1}}{\sqrt{2 \sigma^{2}-2 \operatorname{cov}\left(n_{1}, n_{2}\right)}}\right)
\end{aligned}
$$

\section{B. BER for peak detector}

According to the architecture of peak detector, its bit error probability can be easily derived as

$$
P_{e 2_{-} P P M}=Q\left(\frac{A}{\sqrt{2 N_{0} B \bullet\left[1-\operatorname{sinc}\left(\pi B T_{c}\right)\right]}}\right)
$$

Where, $\boldsymbol{A}$ is the amplitude value of the peak point of the received waveform. That is

$$
A=\max _{t \in T}\left\{r_{1}(t)\right\}=\max _{t \in T}\left\{s(t)+n_{L}(t)\right\}
$$

\section{BER Approximation for ED receiver}

The architecture of square-law receiver (the ED receiver) is different from the Direct-integral-receiver and peak detector, for its nonlinear character, it is difficult to acquire analytic solution.

However, by using Shannon's sampling theorem, one can show that the energy in a finite time interval can be described as a sum of the square of a number of statistically independent Gaussian variables if the noise input is Gaussian and has a flat spectral density over a limited bandwidth [3].

Under this architecture, our analysis is obtained from [4], according to the Fig.3. $\boldsymbol{y}$ can be expressed as

$$
y=\int_{0}^{T_{c}}\left(a_{i} s_{(t)}+n_{L(t)}\right)^{2} d t \approx \frac{1}{T_{s}} \sum_{k=1}^{M}\left(a_{i} s_{k}+n_{k}\right)^{2}
$$

Where, $M$ is the number of samples per component (we finally have $M$ terms to sum over), and generally $S_{k}$ and $n_{k}$, respectively, denote the $k$-th samples of $s(t)$ and $n_{L}(t)$.

It then follows that $\boldsymbol{y}$ satisfies chi-square distribution, with its variance $\sigma_{\kappa}^{2}=1$, non-centrality parameter $\mu=2 \alpha$, $\alpha=E_{b} / N_{0}$ and $M$ degrees of freedom (DOF), $E_{b}$ is the energy of signal $s(t)$. Likewise, for a given $s(t), y$ will be central chi-square distributed. Now, the probability density function (PDF) of $y$ can be expressed as

$$
f_{Y}(y)= \begin{cases}\frac{y^{M / 2-1}}{\sigma_{\kappa}^{M} 2^{M / 2} \Gamma(M / 2)} e^{-\frac{y}{2 \sigma_{\kappa}^{2}}} & a_{i}=0 \\ \frac{1}{2 \sigma_{\kappa}^{2}}\left(\frac{y}{2 \alpha}\right)^{\frac{M-2}{4}} e^{-\frac{2 \gamma+y}{2 \sigma_{\kappa}^{2}}} I_{\frac{M}{2}-1}\left(\frac{\sqrt{2 \alpha y}}{\sigma_{\kappa}^{2}}\right) & a_{i}=1\end{cases}
$$

Where $\Gamma(\bullet)$ is the gamma function and $I_{v}(\bullet)$ is the $v$-th order modified Bessel function of the first kind.

An approximate expression for the probability of bit error over AWGN channels was presented in [3]. Based on the statistics of $y$, when $V$ is the decision threshold, assume the symbol ' 0, '' 1 ' are sent in equal probability, then the error probability $p_{\mathrm{e}}$ can be evaluated as

$$
p_{e}=\frac{\Gamma\left(\frac{M}{2}, \frac{V}{2 \sigma_{\kappa}^{2}}\right)}{2 \Gamma\left(\frac{M}{2}\right)}+\frac{1}{2} Q_{M / 2}\left(\sqrt{\frac{2 \alpha}{\sigma_{\kappa}^{2}}}, \sqrt{\frac{V}{\sigma_{\kappa}^{2}}}\right)
$$

where $\Gamma(\bullet, \bullet)$ is the incomplete gamma function. $Q_{M / 2}(\bullet, \bullet)$ is the generalized Marcum-function [5].

\section{SimULATIONS}

Theoretical curves of direct-integral receiver, peak detector and correlation-receiver and theirs simulation results over the AWGN channel are given in Fig.4. And Fig.5 gives the BER performance comparison between direct-integral receiver and $\mathrm{ED}$, and theirs performance when the time integral interval decreased. In both figures, we adopt the Gaussian pulse with a bandwidth $B$ of $1 \mathrm{GHz}$ as the received signal forms in the simulation.

Fig.4. shows that the simulation results is well agree with the theoretical curves, compare with peak detector the BER performance of Direct-integral receiver is better with a power advantage which is approach to $2.45 \mathrm{~dB}$ with respect to $E_{\mathrm{b}} / N_{0}$ at BER of $10^{-2}$. Compare with a correlation receiver, direct-integral receiver has approximately a power weakness of $3.6 \mathrm{~dB}$ when BER is at $10^{-3}$.

It is of interest to compare a direct-integral receiver with a square-law receiver. This comparison is shown in Fig.5 square-law receiver has approximately a power advantage of $3 \mathrm{~dB}$ when BER is at $10^{-3}$. But under small $E_{\mathrm{b}} / N_{0}$ (less than $6.5 \mathrm{db}$ ), the BER performance of direct-integral receiver is better. Besides, in this simulation for both square-law receiver and direct-integral receiver theirs BER performance deteriorate significantly when the integral time duration is halved.

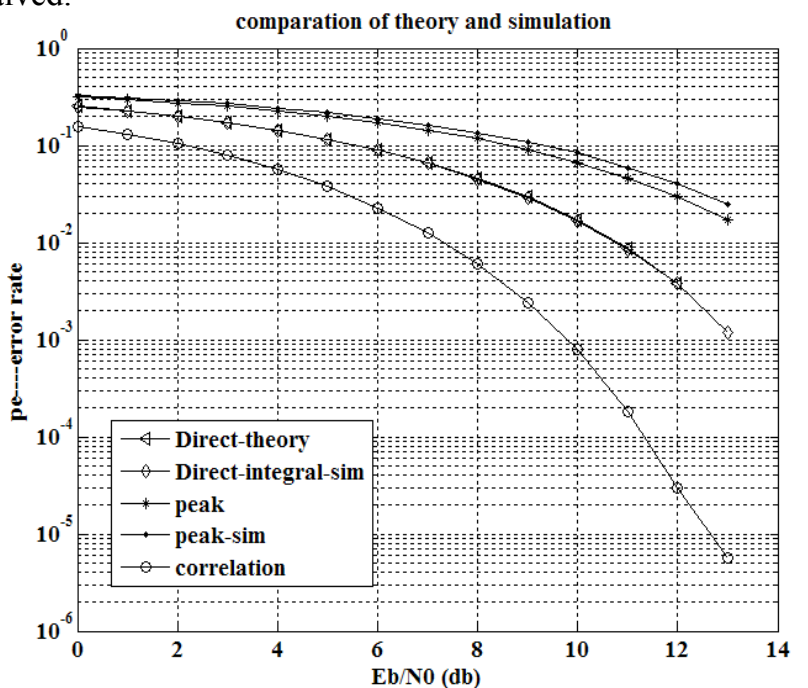

Fig .4 Bit error rates for theoretical and simulation of three kind detectors 


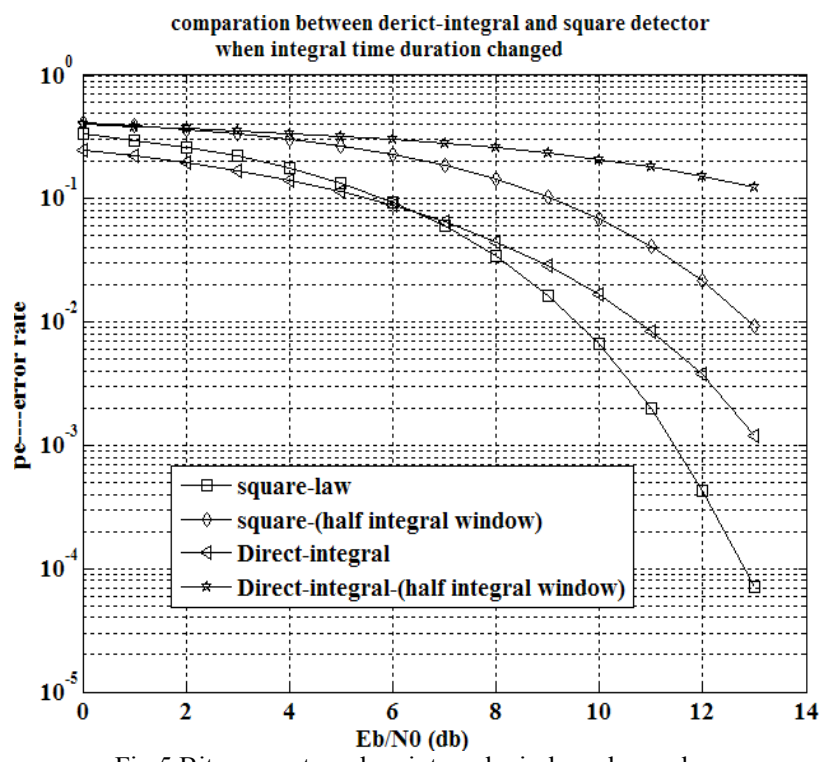

Fig.5 Bit error rates when integral window changed

\section{DISCUSSION AND ANALYSIS}

On the one hand, if the form of a signal to be detected is unknown, it appears appropriate to consider an energy detector as a device for deciding whether or not the signal is present. Since an energy detector does not care about any-thing but the amount of energy in the given observation time, the form of the signal does not affect the conditional probability that a threshold will be exceeded when the signal is present. However, the square-law also increases the effect of noise accumulation, thus the SNR output of the decision variable not increases in the same pace.

The direct-integral receiver has its own merits, first it has better error rate performance than square-law receiver in small $E_{\mathrm{b}} / N_{0}$, its integral module can offset some influence brought by noise while accumulating the effect of signal we wanted, for the integral module has the effect of summarizing, as long as the noise is zero mean Gaussian process, when summed up it remains zero mean Gaussian variable, thus, the SNR output of the decision variable of receiver is little higher compare with the square-law case. Second, direct-integral receiver detectors have different features from the EDs such as the piecewise linear property and the utilization of the integration of signal amplitude for signal detection. Those characteristics deserve further investigation of new signal processing techniques which make it potential for Direct-integral receiver to achieve an appropriate tradeoff between error performance and system complexity compared with EDs.

On the other hand, the BER performance also depends on the integral time duration $T_{c}$ the longer $T_{c}$ is, the better the BER performance is. This is another way of stating the obvious conclusion that the observation time should be as long as possible, for beyond the observation window the SNR of received waveform can not be neglected. Meanwhile
A none-negative signal is better than a signal with both positive and negative peak.

\section{CONCLUSIONS}

The theoretical error performance of Direct-integral receiver and peak detector are studied in this paper. It shows that simulation results in accordance with the theoretical curves. Compared with the ED, the Direct-integral receiver has a little power advantage when $E_{b} / N_{0}$ is small(less than $6.5 \mathrm{db}$ ), whereas the square-law receiver has approximately a power advantage of $3 \mathrm{~dB}$ when BER is at $10^{-3}$. With the time duration of received signal shortened, the BER performance of the detector deteriorated greatly.

In this study, we only focus on the basic detection architecture and signal processing of direct-integral receiver and peak detector. Future works need to be done considering more factors.

\section{ACKNOWLEDGMENT}

This work is supported by National 863 high-tech program of China with No. 2007AA01Z2b3.

\section{REFERENCES}

[1] Witrisal, K., Leus, G., Janssen, G., Pausini, M., Troesch, F., Zasowski, T., Romme, J., "Noncoherent ultra-wideband systems", IEEE Signal Processing Magazine, 2009, 26, (4), pp. 48-66

[2] Yafei Tian; Chenyang Yang;"Noncoherent Multiple-Symbol Detection in Coded Ultra-wideband Communications," Wireless Communications, IEEE Transactions on , vol.7, no.6, pp.2202-2211, June 2008

[3] H.Urkowitz,"Energy detection of unknown deterministic signals"Proc. IEEE, vol. 55, no. 4, pp. 523-531, Apr. 1967.

[4] Fadel F. Digham, Mohamed-Slim Alouini, Marvin K. Simon, " On the Energy Detection of Unknown Signals Over Fading Channels" IEEE IEEE Transactions on communication, vol.55 no.1 january 2007 pp 21-24.

[5] A.H.Nuttall,"Some integrals involving the Q-function," Naval Underwater Syst. Center (NUSC) Tech. Rep., May 1974.

[6] Xiantao Cheng; Yong Guan; , "Mitigation of cross-modulation interference in UWB energy detector receivers," Communications Letters, IEEE, vol.13, no.6, pp.375-377, June 2009

[7] Jinyun Zhang, Orlik, P.V., Sahinoglu, Z., Molisch, A.F., Kinney, P., "UWB Systems for Wireless Sensor Networks", Proceedings of the IEEE, 2009, 97(2), pp. 313-331

[8] Sahin, M.E.; Guvenc, I.; Arslan, H.; , "Optimization of energy detector receivers for UWB systems," Vehicular Technology Conference, 2005. VTC 2005-Spring. 2005 IEEE 61st, vol.2, no., pp. 1386- 1390 Vol. 2, 30 May-1 June 2005

[9] Jorge Moragues, Luis Vergara, Jorge Gosálbez, Ignacio Bosch, "An extended energy detector for non-Gaussian and non-independent noise", Signal Processing, 2009, 89(4), pp. 656-661.

[10] Urkowitz, H.; , "Energy Detection of a Random Process in Colored Gaussian Noise," Aerospace and Electronic Systems, IEEE Transactions on, vol.AES-5, no.2, pp.156-162, March 1969

[11] Urkowitz, H.; , "Energy detection of unknown deterministic signals," Proceedings of the IEEE, vol.55, no.4, pp. 523- 531, April 1967

[12] W. B. Davenport, Jr., W. L. Root, "An Introduction to the Theory of Random Signals and Noise," IEEE Communication Society, Inc., New York, N. Y., 1987.

[13] Na, Y.; Saquib, M.; , "Analysis of the Channel Energy Capture in Ultra-Wideband Transmitted Reference Systems," Communications, IEEE Transactions on , vol.55, no.2, pp.262-265, Feb. 2007 